\title{
RATES OF SURGICAL SITE INFECTION IN CHOLECYSTECTOMY: COMPARISON BETWEEN A UNIVERSITY TEACHING HOSPITAL, MADRID REGION, SPAIN, AND USA RATES
}

\author{
Gil Rodríguez-Caravaca ${ }^{1,2 *}$, Pablo Gil-Yonte ${ }^{3}$, Juan Antonio Del-Moral-Luque ${ }^{1,2}$, \\ Warren Covelé Lucas ${ }^{1,2}$, José María Fernández-Cebrián ${ }^{3}$ And Manuel Durán-Poveda ${ }^{2}$ \\ ${ }^{1}$ Department of Preventive Medicine and Public Health, Hospital Universitario Fundación Alcorcón; ${ }^{2}$ Department of \\ Medicine, Surgery, Preventive Medicine and Public Health, Universidad Rey Juan Carlos; ${ }^{3}$ Department of Surgery, \\ Hospital Universitario Fundación Alcorcón. Madrid, Spain
}

\begin{abstract}
Background: There are many factors that can influence surgical site infections (SSI) in cholecystectomies. Incidence of cholecystectomy SSI was studied and compared with the incidence in Madrid Region, Spain, and the United States. Methods: A prospective cohort study was conducted which included all patients who underwent gallbladder surgery for 5 consecutive years, at the Alcorcón Foundation University Teaching Hospital. SSI incidence rate was calculated. An association between risk factors and SSI incidence was assessed with the relative risk (RR). Infection rates were compared to those in the Madrid Region and to the overall Spanish and United States rates using the standardized infection ratio (SIR). Results: The study included 1532 patients. Cumulative overall SSI was $1.96 \%$ (95\% confidence interval [CI]: 1.3-2.7). The SIR was 0.89 with respect to the Madrid Region, 0.77 with respect to Spain's rate, and 1.77 with respect to the United States' rate. A laparoscopic route protected against infection ( $R R=0.43 ; 95 \% \mathrm{Cl}$ : 0.2-0.9). Razor shaving in surgical preparation, duration of surgery, and neoplasm increased SSI incidence. Conclusions: SSI incidence rates among cholecystectomized patients at our hospital are higher than rates in the United States. A laparoscopic route protected against SSI.
\end{abstract}

Key words: Surgical wound infection, Cohort studies, Incidence, Cholecystectomy.

\section{INTRODUCTION}

A nosocomial (i.e., hospital-acquired) infection is defined as any infection, which is not present or incubated at the time of admission, and occurs $48 \mathrm{~h}$ or more after

\section{Corresponding author:}

*Gil Rodríguez-Caravaca

Servicio de Medicina Preventiva y Salud Pública

Hospital Universitario Fundación Alcorcón

Budapest, 1

C.P. 28922, Alcorcón, Madrid, Spain

E-mail: grodriguez@fhalcorcon.es admission $^{1}$. In the latest review issued by the Centers for Disease Control and Prevention (CDC, 2008), such infections have come to be known as "healthcare-associated infections" 2 and affect $5 \%$ to $10 \%$ of hospitalized patients, thus posing an important public health problem ${ }^{3,4}$.
Received for publication: 31-03-2017

Accepted for publication: 20-06-2017

doi: $10.24875 / R I C .17002197$ 
Surgical site infection (SSI) is the most frequent complication among patients undergoing cholecystectomy $y^{5,6}$ and is considered by the World Health Organization as a health care indicator, not only because of its socio-healthcare and economic costs but also because of its associated morbidity and mortality ${ }^{7}$. The SSI rate in cholecystectomy ranges from $0.5 \%$ to $5 \%$, depending on the type of hospital, surgical unit assessed, and infection surveillance system used. According to the multicenter Clinical Indicators of Continuous Quality Improvement Indicadores Clínicos de Mejora Continua de Calidad (INCLIMECC) study ${ }^{8}$, SSI rates for cholecystectomy are $3.2 \%$ in the Madrid Autonomous Region (Comunidad Autónoma de Madrid), and $3.6 \%$ in Spain as a whole ${ }^{8}$. Both of these figures are higher than the comparable CDC rates ${ }^{9}$. The main SSI risk factors in cholecystectomies are comorbidity, duration of surgery, degree of contamination of the surgical intervention, adequacy of surgical preparation, appropriateness of antibiotic prophylaxis, quality of the surgical technique, and type of surgery, i.e., open or laparoscopic ${ }^{10-12}$. This study's principal aim was to first, study the risk factors linked to cholecystectomy-related SSI incidence; and second, to compare this incidence to rates in the vicinity of the Madrid Region, rates at other hospitals across Spain, and figures issued by the CDC (USA).

\section{MATERIALS AND METHODS}

\section{Patients and study design}

A prospective cohort study at the Alcorcón Foundation University Teaching Hospital (AFUTH) was conducted. The study included all patients who underwent gallbladder surgery (open and laparoscopic) from 1 July 2009 to 30 June 2016. The criteria for exclusion were: (1) Being on antibiotic treatment, or (2) having a diagnosis of infection at the date of the intervention. The sample size was estimated on the basis of an expected SSI incidence of 3\%, a $95 \%$ confidence level, a precision of $1 \%$, and losses to follow-up of $10 \%$. We, thus, estimated that it would be necessary to study 1237 patients. The CDC's SSI criteria were taken into account, with regard to the depth of the infection being divided into superficial, deep incisional, and organ-space ${ }^{13,14}$. The study was approved by the AFUTH Ethics Committee and Research Board.
The task of data collection and recording was undertaken by the medical staff of the Preventive Medicine Unit, i.e., physicians and nurses, based on information drawn from electronic medical records, microbiologic cultures, and case comments made by the clinicians and nurses who attended to the patients. The diagnosis of SSI was, in all cases, made jointly by a preventive medicine physician and a surgeon. Data recording, management, and analysis were performed using the INCLIMECC program ${ }^{8}$. SSI surveillance was maintained for 30 days post-surgery, and during their stay in hospital, patients were evaluated daily. After discharge, patients were actively followed up on, by reviewing their electronic medical records, monitoring their progress, and their attendance at hospital outpatient facilities, at emergency wards, or in primary care.

The following variables were analyzed: Sex, age, and patient comorbidity (malnutrition, coma, kidney failure, diabetes mellitus, neoplasm - cancer regardless the location -, chronic obstructive pulmonary diseases, cirrhosis, obesity, injection drug abuse, and neutropenia -neutrophil count $<0.5 \times 10^{9} / \mathrm{L}$ ); use of drains, pre-operative razor shaving, transfusion, elective/emergency surgery, open/laparoscopic surgery, duration of surgery, American Society of Anesthesiologists (ASA) risk score, degree of contamination of surgery (clean, clean-contaminated, contaminated, and dirty), presence or absence of SSI, microorganisms implicated, adequacy or inadequacy of surgical preparation (pre-operative chlorhexidinebased body showers/baths and mouth rinses non-administered were considered inadequate), administration or non-administration of antibiotic prophylaxis, and appropriateness of prophylaxis (time of initiation, antibiotic, route, dose, and duration) according to the hospital's protocol (inadequacy if any of the items failed). According to the antibiotic prophylaxis protocol, it was only administered in high-risk patients (aged over 70, choledocholithiasis, and prior instrumentation of bile ducts). Antibiotic prophylaxis consisted of $2 \mathrm{~g}$ of intravenous amoxicillin clavulanate administered $30-60 \mathrm{~min}$ before surgery. Patients allergic to beta-lactam received $80 \mathrm{mg}$ of intravenous gentamicin administered 30-60 min before surgery.

A descriptive study was performed to establish patients' sociodemographic and clinical characteristics. 
The sample was categorized according to the $\mathrm{Na}$ tional Nosocomial Infections Surveillance (NNIS) index (CDC), which has a value ranging from 0 to 3 and is calculated by adding the ASA score ( 1 point if $>2$ ), degree of contamination of surgery ( 1 point if contaminated or dirty), and duration of surgery ( 1 point if higher than the $75^{\text {th }}$ percentile). Laparoscopic cholecystectomy subtracted one point to the final NNIS index. Thus, laparoscopic cholecystectomies with NNIS 0 were named as NNIS M.

Infection rates, both overall and stratified according to the NNIS index, were calculated. In addition, the average lengths of pre-operative and total hospital stays were also calculated.

\section{Statistical analysis}

We compared our AFUTH data to data for the Madrid Region, Spain, and the USA using the indirect standardization method. The standardized infection ratio (SIR) is the observed number of infections divided by the expected ones and is interpreted as a relative risk (RR). The use of SIR allows a global comparison and quantifies the real existing difference better than the SSI rates because the number of infections is corrected for the different risk categories $^{10}$.

A univariate and a multivariate logistic regression analysis were used to find independently associated risk factors for SSI. Qualitative variables were compared using Pearson's $\chi^{2}$ test or Fisher's nonparametric exact test. Quantitative variables were analyzed using the Student's $t$-test or the Mann-Whitney test.

A multivariate logistic regression model was built following backward stepwise selection. This procedure provides a fast and effective means to screen a large number of variables, and they were introduced in the models when $p<0.2$ in the univariate analysis ${ }^{11}$. The association between risk factors and SSI incidence was assessed by the RR, and the fitness of the model stated.

Statistical analysis was performed, with hypothesis testing based on a two-tailed test of significance and an alpha level of $p<0.05$.

\section{RESULTS}

The study included a total of 1532 patients, 618 men $(40.3 \%)$ and 914 women $(59.7 \%)(p<0.05)$; the overall median age was 62 years (Interquartile range $[I Q R]=45-73), 63$ years $(I Q R=46-72)$ for men and 61 years $(I Q R=44-74)$ for women $(p>0.05)$. A total of $86.4 \%$ (1323) of interventions were elective, and cholecystectomies were laparotomic in 351 (22.9\%), and laparoscopic in 1,181 cases $(77.1 \%)$. The percentage of patients with laparoscopic surgery converted to open surgery was $12 \%$. Prophylactic therapy with antibiotics was indicated in 743 patients $(48.5 \%)$. The mean overall stay was 5.4 days, with a mean pre-operative stay of 1.1 day, and a mean post-operative stay of 4.3 days. Among patients with $\mathrm{SSI}$, the mean pre-operative stay was 2.5 days, and the mean post-operative stay was 22 days. The general descriptive data of the patients are shown in table 1.

Cumulative overall SSI incidence was 1.96\% (95\% confidence interval $[\mathrm{Cl}]: 1.3-2.7$ ), with a total of 30 surgical infections. The number of patients studied decreased according to the NNIS index, namely, NNIS M, 52.4\% (388 patients), NNIS 0, 24.6\% (183 patients), NNIS 1, $12.9 \%$ (96 patients), NNIS 2, $6.8 \%$ (50 patients), and NNIS 3, 3.3\% (26 patients). The percentage of infections classified by depth was as follows: $1.0 \%$ superficial incisional, $0.3 \%$ deep incisional, and $0.66 \%$ organ-space. The SIR for the Madrid Region was $0.89,0.77$ with respect to Spain's national rate, and 1.77 with respect to the US NHSN/CDC rate. The frequency of SSI was seen to increase progressively (from $0.8 \%$ to $8 \%$ ) as the NNIS index rose from $\mathrm{M}$ to 3 .

A total of $90 \%$ (15 patients) of patients with infection had a positive microbiologic culture: 8 of these patients had polymicrobial infections, with two (6) or more (2) microorganisms being isolated in cultures. The most frequent microorganisms implicated in SSI were Escherichia coli (47\%), Klebsiella pneumoniae (13\%), Enterococcus faecium (10\%), and Staphylococcus aureus (10\%) (Fig. 1).

The intrinsic SSI risk factors were observed to be diabetes mellitus (14.9\%), obesity (13.8\%), neoplasm $(7.5 \%)$, chronic obstructive pulmonary disease 
Table 1 . Characteristics of patients $(n=1532)$ undergoing cholecystectomy

\begin{tabular}{|c|c|}
\hline \multicolumn{2}{|l|}{ Characteristic } \\
\hline Mean age $\pm S D^{*}$ (years) & $58.9 \pm 16.9$ \\
\hline \multicolumn{2}{|l|}{ Gender n (\%) } \\
\hline Male & $618(40.3)$ \\
\hline Female & $914(59.7)$ \\
\hline \multicolumn{2}{|l|}{ ASA score $\mathrm{n}(\%)$} \\
\hline I & $267(17.4)$ \\
\hline$\|$ & $981(64.0)$ \\
\hline III & $253(16.5)$ \\
\hline IV & $32(2.1)$ \\
\hline \multicolumn{2}{|c|}{ Most frequent clinical diagnosis (ICD-9 classification) n (\%) } \\
\hline 574.10 cholelithiasis with cholecystitis & $980(64.0)$ \\
\hline 574.00 cholelithiasis with acute cholecystitis & $251(16.4)$ \\
\hline 574.20 cholelithiasis & $141(9.2)$ \\
\hline 575.0 acute cholecystitis & $27(1.8)$ \\
\hline \multicolumn{2}{|l|}{ Surgical intervention $\mathrm{n}(\%)$} \\
\hline Emergency & $208(13.6)$ \\
\hline Non-emergency & $1323(86.4)$ \\
\hline \multicolumn{2}{|c|}{ Most frequent surgical procedures (according to the ICD-9 classification) $n(\%)$} \\
\hline 51.23 laparoscopic cholecystectomy & $1181(77.1)$ \\
\hline 51.22 laparotomic cholecystectomy & $351(22.9)$ \\
\hline \multicolumn{2}{|l|}{ Duration of surgery* } \\
\hline Mean $(\min ) \pm S D$ & $83(50.1)$ \\
\hline$>75^{\text {th }}$ percentile $(>95 \mathrm{~min}) \mathrm{n}(\%)$ & $393(25.6)$ \\
\hline$<75^{\text {th }}$ percentile $(<95 \mathrm{~min}) \mathrm{n}(\%)$ & $1139(74.4)$ \\
\hline \multicolumn{2}{|l|}{ Pre-operative preparation n (\%) } \\
\hline Correct & $1197(78.2)$ \\
\hline Preparation not performed & $164(10.6)$ \\
\hline No clinical reports & $76(5.0)$ \\
\hline No oral antiseptic & $93(6.1)$ \\
\hline No body wash & $2(0.1)$ \\
\hline \multicolumn{2}{|l|}{ Antibiotic prophylaxis n=743 (\%) } \\
\hline Appropriate prophylaxis & $549(73.9)$ \\
\hline Inappropriate prophylaxis & $194(26.1)$ \\
\hline Duration & $8(1.1)$ \\
\hline Choice & $135(18.2)$ \\
\hline Time & $51(6.9)$ \\
\hline \multicolumn{2}{|l|}{ NNIS index** $\mathrm{n}(\%)$} \\
\hline M & $805(52.4)$ \\
\hline 0 & $375(24.6)$ \\
\hline 1 & $197(12.9)$ \\
\hline 2 & $105(06.8)$ \\
\hline 3 & $50(3.3)$ \\
\hline Mean pre-operative hospital stay (days) (SD)* & $1.1(2.8)$ \\
\hline Median length of stay (days) (range) & $12.7(49)$ \\
\hline
\end{tabular}

*Continuous variables are expressed with mean and SD.

**NNIS: National Nosocomial Infection Surveillance, CDC, USA.

CDC: Centers for Disease Control and Prevention; SD: Standard deviation. 
Figure 1. Etiology (\%) of surgical site infection $(n=30)$.

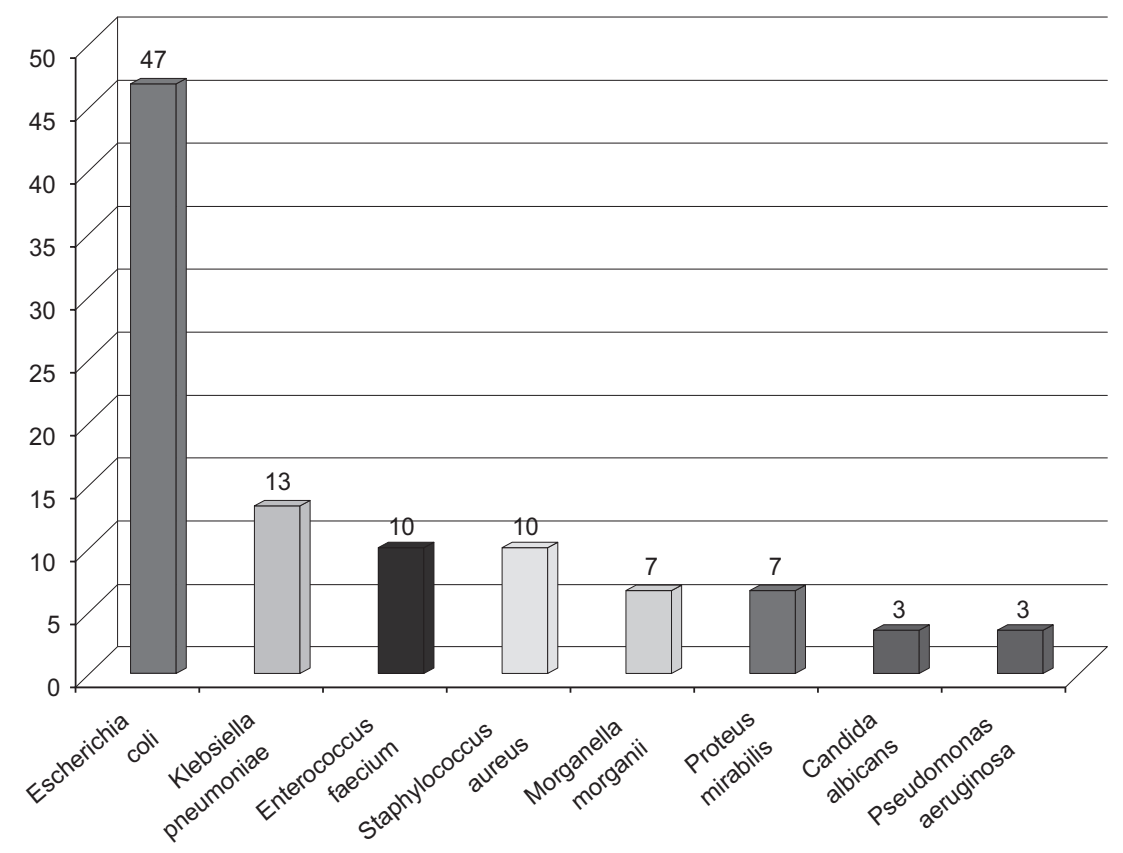

(3.5\%), kidney failure $(2.2 \%)$, cirrhosis $(0.1 \%)$, and neutropenia (0.1\%).

While overall adequacy of antibiotic prophylaxis was $73.9 \%(95 \% \mathrm{Cl}: 69 \%-75 \%)$, the greatest degree of inadequacy of the protocol was observed in the choice of antibiotic (18.2\%; 95\% Cl: 17-20), followed by the time of initiation of prophylaxis (6.9\%; $95 \% \mathrm{Cl}: 6-9)$, and the duration of prophylaxis (1.1\%; $95 \%$ $\mathrm{Cl}$ : 0.1-2.2). Adequacy was $100 \%$ for both dosage and route of administration. Surgical pre-operative preparation was neither performed nor registered in $15.6 \%$ of patients, and it was adequate in $78.2 \%$ of them. Pre-operative body wash and antiseptic mouth rinses were not used in $0.1 \%$ and $6.1 \%$ of cases, respectively. Razor shaving before surgery was performed on $63 \%$ of patients.

In the univariate analysis, a significant association was observed between SSI and dirty surgery, duration higher than the $75^{\text {th }}$ percentile, ASA risk value more than 2, neoplasm, razor shaving, placement of drains, emergency cholecystectomy, and laparoscopic surgery (Table 2).

The multivariate analysis showed a significant association between SSI and the use of laparoscopic route $(\mathrm{RR}=0.43 ; 95 \% \mathrm{Cl}: 0.2-0.9)$, razor shaving in surgical preparation $(\mathrm{RR}=2.9 ; 95 \% \mathrm{Cl}: 1.3-6.3)$, duration of surgery higher than the $75^{\text {th }}$ percentile $(R R=2.6$; $95 \% \mathrm{Cl}: 1.2-5.7)$, and presence of neoplasm ( $R R=8.3$; 95\% Cl: 3.6-19.1) (Table 3).

\section{DISCUSSION}

This study observed an SSI incidence of $1.96 \%$, which is lower than the mean for the Madrid Region and Spain overall but higher than the figures issued by the NSHN/CDC ${ }^{9}$. The SIR was 0.89 with respect to the incidence for the Madrid Region, 0.77 with respect to that for the country as a whole ${ }^{8}$, and 1.77 with respect to the NHSN/CDC figures ${ }^{12}$. One of the possible reasons for this difference in rates is that SSI surveillance at the AFUTH is conducted for the maximum incubation period of 30 days after discharge, by reviewing the clinical progress of patients receiving attention for SSI at the hospital's emergency ward and outpatient facilities as well as in primary care, based on our electronic medical records (Selene ${ }^{\circledR}$, $\mathrm{HORUS}^{\circledR}$ ).

In their study for the American College of Surgeons National Surgical Quality Improvement Program (ACS NSQIP) covering 65,511 cholecystectomized patients from 2005 to 2008, Ingraham et al. ${ }^{15}$ observed an SSI 
Table 2. Univariate analysis ( $\mathrm{n}=1532)$ of risk factors for surgical site infection in cholecystectomy

\begin{tabular}{|c|c|c|c|c|c|c|}
\hline Characteristics & Total (\%) & SSI (\%) & Non SSI (\%) & $\mathrm{RR}_{\mathrm{c}}$ & $95 \% \mathrm{Cl}$ & $\mathrm{p}$ \\
\hline Diabetes mellitus & $228(14.9)$ & $4(1.8)$ & $224(98.2)$ & 0.9 & $0.3-2.5$ & 0.81 \\
\hline Neoplasm & $115(7.5)$ & $12(10.4)$ & $103(89.6)$ & 8.2 & $4.1-16$ & 0.000 \\
\hline Sex: Male & $618(40.3)$ & $17(2.8)$ & $601(97.2)$ & 0.5 & $0.2-1.1$ & 0.06 \\
\hline Laparoscopy & $1181(77.1)$ & $15(1.3)$ & $1166(98.7)$ & 0.3 & $0.2-0.6$ & 0.004 \\
\hline Razor shaving & $371(24.2)$ & $14(3.8)$ & $357(96.2)$ & 2.7 & $1.3-5.5$ & 0.004 \\
\hline Inadequate prophylaxis & $194(26.2)$ & $3(1.5)$ & $191(98.5)$ & 1.1 & $0.3-4.0$ & 0.8 \\
\hline Inadequate preparation & 335 (21.9) & $9(2.7)$ & $326(97.3)$ & 1.3 & $0.6-2.9$ & 0.5 \\
\hline Emergency intervention & $209(13.6)$ & $11(5.3)$ & $198(94.7)$ & 3.7 & $1.8-7.6$ & 0.002 \\
\hline Dirty surgery & $172(11.2)$ & $7(4.1)$ & $165(95.9)$ & 2.2 & $1.0-5.5$ & 0.04 \\
\hline Surgery duration >p75 & $172(11.2)$ & $7(4.1)$ & $332(94.9)$ & 5.1 & $2.5-10$ & 0.000 \\
\hline ASA score $>2$ & $285(18.6)$ & $13(4.6)$ & $272(95.4)$ & 3.5 & $1.7-7.1$ & 0.002 \\
\hline Drains & $451(29.4)$ & $20(4.4)$ & $431(95.6)$ & 4.8 & $2.3-10$ & 0.000 \\
\hline Age $>65$ years & $852(55.6)$ & $18(2.1)$ & $834(97.9)$ & 0.8 & $0.3-1.6$ & 0.48 \\
\hline Obesity & $211(13.8)$ & $0(0.0)$ & $211(100)$ & 0.3 & $0.1-1.6$ & 0.21 \\
\hline COPD & $53(3.5)$ & $2(3.8)$ & $51(96.2)$ & 2.0 & $0.5-8.2$ & 0.33 \\
\hline
\end{tabular}

SSI: Surgical site infection; RRc: Crude relative risk; COPD: Chronic obstructive pulmonary disease; Cl: Confidence interval.

Table 3. Multivariate analysis $(n=1532)$ of associated risk factors for surgical site infection in cholecystectomy

\begin{tabular}{lccc}
\hline Characteristics & RR $_{\text {adjusted }}$ & $95 \% \mathrm{Cl}$ & $\mathrm{p}$ \\
\hline Laparoscopy & 0.43 & $0.1-0.9$ & 0.01 \\
Razor shaving & 2.9 & $1.1-5.3$ & 0.002 \\
Duration of surgery $>$ p75 & 2.6 & $1.2-4.7$ & 0.005 \\
Neoplasm & 8.3 & $2.6-13.6$ & 0.001 \\
\hline
\end{tabular}

RR: Relative risk; $\mathrm{Cl}$ : Confidence interval.

rate of $2.0 \%$, a figure slightly higher than ours. The study also observed a significantly lower SSI incidence among patients who underwent laparoscopic surgery than among those who underwent open surgery (1.3\% in laparoscopic and $4.3 \%$ in open surgery at the AFUTH vs. $1.3 \%$ in laparoscopic surgery, and $8.4 \%$ in laparotomy in Ingraham's series).

As in other studies ${ }^{16,17}$, the microorganism most frequently isolated in cholecystectomized patients with SSI at AFUTH hospital was E. coli (47\%), followed by K. pneumoniae (13\%), and E. faecium (10\%).

One factor shown to be significantly associated with $\mathrm{SSI}$, a finding that has also been reported by other studies, was pre-operative razor shaving. Indeed, the elimination of body hair by shaving during surgical preparation causes micro lesions in the skin that facilitate colonization of the surgical wound by bacteria, thereby increasing the risk of SSI. Removal of body hair should thus be avoided if possible or where necessary, should be done using an electric or battery-operated clipper/razor with a reusable head, as close as possible to the starting time of the operation, and at a site outside the operating theater area ${ }^{18}$.

The sole intrinsic risk factor found to be significantly associated with a higher incidence of SSI among patients undergoing cholecystectomy was the presence of related neoplasm. Factors found to be significant in other studies, such as presence of diabetes mellitus, chronic obstructive pulmonary disease, obesity or advanced age, showed no association with SSI in this study's sample ${ }^{19-23}$.

Another important factor to be borne in mind, according to the data of this study, is the mean overall stay of cholecystectomized patients, which in our case was 5.4 days, versus the mean of 6.8 days for the Madrid Region $^{24}$. This mean stay was, likewise, considerably shorter than the Spanish national mean of 10.4 days, though it is nonetheless, higher than those reported for the USA. If this is broken down into mean preoperative and post-operative stays, the AFUTH figures will be seen to be lower than the national ones (1.1 vs. 3.6 days in pre-operative stay, and 4.3 vs. 6.4 days in post-operative stay). If this is further broken down into mean pre-operative and post-operative 
stays in cases with SSI, the AFUTH figures will again be seen to be lower than those at a national level in terms of pre-operative stay ( 2.5 vs. 5.3 days) while being similar in terms of post-operative stay ( $22.0 \mathrm{vs.}$ 21.1 days). It is reasonable to surmise that a longer pre-operative stay would have repercussions in the form of higher SSI incidence due to the risks associated with hospitalization, and also that a higher nationwide SSI incidence in cholecystectomies would be reflected in a parallel increase in mean post-operative stay ${ }^{25-27}$

Another key group of SSI risk factors highlighted by literature are the so-called extrinsic risk factors. Of these, the following warrant special mention, namely, degree of contamination of the surgical intervention, duration of surgery, and ASA risk ${ }^{28}$. By combining all three, we obtained the NNIS Index, which is scored up to 3. When the association between SSI and extrinsic risk factors was explored by us using univariate and multivariate analyses, only duration of surgery higher than the $75^{\text {th }}$ percentile was shown to be significantly associated with SSI by both analyses. In contrast, an ASA risk of over 2 and the fact of surgery being regarded as contaminated or dirty solely displayed a significant association with SSI in the univariate analysis, a finding that was not, however, corroborated in the multivariate analysis. Indeed, it must be stressed that duration higher than the $75^{\text {th }}$ percentile is an extrinsic risk factor which is frequently cited as being significantly associated with SSI in cholecystectomy in SSI studies ${ }^{21}$. The $75^{\text {th }}$ percentile duration at the AFUTH was found to be 95 min versus $125 \mathrm{~min}$ at a national level ${ }^{8}$. In other studies, an ASA risk of over 2 and, in some cases, the fact of surgery being contaminated or dirty have also been found to be significant ${ }^{29}$. In this study, in line with the studies by Culver et al. ${ }^{30}$, SSI incidence was observed to increase in parallel to the NNIS index.

Unlike other studies ${ }^{21}$, this study failed to observe a significant association between the surgeon and SSI, or between the operating theater and SSI.

Appropriateness of antibiotic prophylaxis showed no significant association with SSI incidence among patients undergoing cholecystectomy, in line with most studies on the topic ${ }^{31,32}$. Overall appropriateness of antibiotic prophylaxis was $73.9 \%$, a figure slightly higher than the mean for hospitals in the Madrid
Region (71\%), though there is still considerable room for improvement, particularly when it comes to the choice of antibiotic ${ }^{24}$. This study's adequacy of surgical preparation (78.2\%) similarly failed to show a significant association with $\mathrm{SSI}$, though here again, there is room for improvement.

There are a number of nosocomial infection surveillance systems, but those considered to benchmark are incidence studies such as the one implemented at the AFUTH. In addition to ascertaining SSI incidence rates, these types of studies allow for comparisons with regional, national, and international standards, and for the study of related risk factors, both intrinsic and extrinsic.

Among our study's limitations, it should be pointed out that there are some factors covered in other studies, which could influence the SSI rate but which we did not assess, e.g., rupture of the gallbladder, presence or absence of biliary colic in the 30 days preceding surgery, and certain echographic, or serologic findings.

In conclusion, it should be stressed that, albeit higher than the United States rates published by the NHSN/CDC, the SSI incidence rates among cholecystectomized patients at the AFUTH are appreciably lower than the means for the Madrid Region and Spain as a whole. Our patients are clinically followed up for 30 days post-discharge, and there are a well-protocolized infection surveillance and control system in place, which yields acceptable infection rates. Even so, these could be improved, and all the resources at our disposal should be deployed, to this end.

\section{ACKNOWLEDGMENTS}

The authors thank the European Regional Development Fund (ERDF) and the Health Research Fund (Fondo de Inveslgaciones Sanitarias/FIS) for funding this work with the research projects $\mathrm{PI} 11 / 01272$ and PI14/01136.

\section{REFERENCES}

1. Horan TC, Gaynes RP, Martone WJ, Jarvis WR, Emori TG. CDC definitions of nosocomial surgical site infections, 1992: A modification of CDC definitions of surgical wound infections. Am J Infect Control. 1992;20:271-4. 
2. Horan TC, Andrus M, Dudeck MA CDC/NHSN surveillance definition of health care-associated infection and criteria for specific types of infections in the acute care setting. Am J Infect Control. 2008;36:309-32.

3. Klevens RM, Edwards JR, Richards CL Jr, et al. Estimating health care-associated infections and deaths in U.S. Hospitals, 2002. Public Health Rep. 2007;122:160-6.

4. Awad SS. Adherence to surgical care improvement project measures and post-operative surgical site infections. Surg Infect (Larchmt). 2012;13:234-7.

5. Magill SS, Hellinger W, Cohen J, et al. Prevalence of healthcareassociated infections in acute care hospitals in Jacksonville, Florida. Infect Control Hosp Epidemiol. 2012;33:283-91.

6. Rosenthal VD, Richtmann R, Singh S, et al. Surgical site infections, International Nosocomial Infection Control Consortium (INICC) report, data summary of 30 countries, 2005-2010. Infect Control Hosp Epidemiol. 2013;34:597-604.

7. Brazzelli M, Cruickshank $M$, Kilonzo $M$, et al. Clinical effectiveness and cost-effectiveness of cholecystectomy compared with observation/conservative management for preventing recurrent symptoms and complications in adults presenting with uncomplicated symptomatic gallstones or cholecystitis: A systematic review and economic evaluation. Health Technol Assess. 2014;18:1-101, v-vi.

8. Díaz-Agero Pérez C, Robustillo Rodela A, Pita López MJ, López Fresneña N, Monge Jodrá V; Quality Control Indicator Working Group. Surgical wound infection rates in Spain: Data summary, January 1997 through June 2012. Am J Infect Control. 2014;42:521-4.

9. Edwards JR, Peterson KD, Mu Y, et al. National Healthcare Safety Network (NHSN) report: Data summary for 2006 through 2008, issued December 2009. Am J Infect Control. 2009. 37:783-805.

10. Tang ZY, Guo EQ, Yan HC, Xie QP, Lian JA, Wu YL. Risk factors for infectious morbidity in gallbladder cancer patients treated surgically. Hepatogastroenterology. 2007;54:22-7

11. Gaynes RP, Culver DH, Horan TC, Edwards JR, Richards C, Tolson JS. The national nocomial infections surveillance system. Surgical site infection (SSI) rates in the United States, 1992-1998: The national nosocomial infections surveillance system basic SSI risk index. Clin Infect Dis. 2001;33 (S3):69-77.

12. Mu Y, Edwards JR, Horan TC, Berrios-Torres SI, Fridkin SK. Improving risk-adjusted measures of surgical site infection for the national healthcare safety network. Infect Control Hosp Epidemiol. 2011;32:970-86

13. Friedman ND, Kaye KS, Stout JE, et al. Health care-associated bloodstream infections in adults: A reason to change the accepted definition of community-acquired infections. Ann Intern Med. 2002;137:791-7.

14. Jarvis WR. Benchmarking for prevention: The centers for disease control and prevention's National Nosocomial Infections Surveillance (NNIS) system experience. Infection. 2003;31 Suppl 2:44-8.

15. Ingraham AM, Cohen ME, Ko CY, Hall BL. A current profile and assessment of North American cholecystectomy: Results from the American college of surgeons national surgical quality improvement program. J Am Coll Surg. 2010;211:176-86.
16. den Hoed PT, Boelhouwer RU, Veen HF, Hop WC, Bruining HA. Infections and bacteriological data after laparoscopic and open gallbladder surgery. J Hosp Infect. 1998;39:27-37.

17. Garibaldi RA, Skolnick D, Maglio S, et al. Postcholecystectomy wound infection. The impact of prophylactic antibiotics on the epidemiology of infections. Ann Surg. 1986;204:650-4.

18. Niël-Weise BS, Wille JC, van den Broek PJ. Hair removal policies in clean surgery: Systematic review of randomized, controlled trials. Infect Control Hosp Epidemiol. 2005;26:923-8.

19. Yildiz B, Abbasoglu O, Tirnaksiz B, Hamaloglu E, Ozdemir A, Sayek I. Determinants of postoperative infection after laparoscopic cholecystectomy. Hepatogastroenterology. 2009;56: 589-92.

20. Tocchi A, Lepre L, Costa G, Liotta G, Mazzoni G, Maggiolini F. The need for antibiotic prophylaxis in elective laparoscopic cholecystectomy: A prospective randomized study. Arch Surg. 2000;135:67-70

21. Giger UF, Michel JM, Opitz I, et al. Risk factors for perioperative complications in patients undergoing laparoscopic cholecystectomy: Analysis of 22,953 consecutive cases from the Swiss Association of Laparoscopic and Thoracoscopic Surgery database. J Am Coll Surg. 2006;203:723-8.

22. Sorensen LT, Karlsmark T, Gottrup F. Abstinence from smoking reduces incisional wound infection: $A$ randomized controlled trial. Ann Surg. 2003;238:1-5.

23. Thomsen T, Tønnesen H, Møller AM. Effect of preoperative smoking cessation interventions on postoperative complications and smoking cessation. Br J Surg. 2009;96:451-61.

24. Díaz-Agero-Pérez C, Pita-López MJ, Robustillo-Rodela $A$ Figuerola-Tejerina A, Monge-Jodrá V; Grupo de Trabajo INCLIMECC de la Comunidad de Madrid. Assessment of the surgical site infection in 14 hospitals of the Madrid Region: An incidence study. Enferm Infecc Microbiol Clin. 2011;29:257-62.

25. Mishriki SF, Law DJ, Jeffery PJ. Factors affecting the incidence of postoperative wound infection. J Hosp Infect. 1990;16:223-30.

26. Cruse PJ, Foord R. A five-year prospective study of 23,649 surgical wounds. Arch Surg. 1973;107:206-10.

27. Ortíz-Aguirre AR, Vargas MH, Hernández-Santiago JL, Munguía $C$. Inverse relationship between age and pulmonary function decline in the postoperative period of laparoscopic cholecystectomy. Rev Invest Clin. 2005;57:420-4.

28. Tang $\mathrm{R}$, Chen $\mathrm{HH}$, Wang $\mathrm{YL}$, et al. Risk factors for surgical site infection after elective resection of the colon and rectum: $A$ single-center prospective study of 2,089 consecutive patients. Ann Surg. 2001;234:181-9.

29. Guzmán-Valdivia G. Routine administration of antibiotics to patients suffering accidental gallbladder perforation during laparoscopic cholecystectomy is not necessary. Surg Laparosc Endosc Percutan Tech. 2008;18:547-50.

30. Culver DH, Horan TC, Gaunes RP, et al. National Nosocomia infection surveillance system. Surgical wound infection rates by wound class, operative procedure, and patient risk index. Am J Med. 1991;91:152-7.

31. Zhou H, Zhang J, Wang Q, Hu Z. Meta-analysis: Antibiotic prophylaxis in elective laparoscopic cholecystectomy. Aliment Pharmacol Ther. 2009;29:1086-95

32. Choudhary A, Bechtold ML, Puli SR, Othman MO, Roy PK. Role of prophylactic antibiotics in laparoscopic cholecystectomy: A meta-analysis. J Gastrointest Surg. 2008;12:1847-53. 